\title{
Perspectives of Nurses and Midwives on Determinants of Contemporary Image of Nursing and Midwifery Professions in Ghana: A Mixed Method Study
}

\section{Gladys Dzansi}

University of Ghana School of Nursing

JOSEPHINE KYEI ( $\nabla$ Jmkyei@ug.edu.gh )

University of Ghana School of Nursing https://orcid.org/0000-0003-3633-366X

Lillian Akorfa Ohene

University of Ghana School of Nursing

Angela Kwartemaa Acheampong

Wisconsin International University College Ghana

Charles Ampong Adjei

University of Ghana School of Nursing

\section{Samuel Adjorlolo}

University of Ghana School of Nursing

Isabella Naana Akyaa Asante

Healthcare Services

\section{Philomena Woolley}

Nurses and Midwives Council

\section{Felix Nyante}

Nurses and Midwives Council

\section{Lydia Aziato}

University of Ghana

\section{Research article}

Keywords: Nursing, Midwifery, image, professional, mixed method, Ghana

Posted Date: November 18th, 2020

DOl: https://doi.org/10.21203/rs.3.rs-105362/v1

License: (9) This work is licensed under a Creative Commons Attribution 4.0 International License. 
Page $2 / 26$ 


\section{Abstract}

Background: Nursing and midwifery image has endured positive and negative stereotypes in transition from vocation to a recognised profession and a discipline. Nevertheless, concerns about professional image still persist and factors influencing contemporary image of nursing and midwifery are least understood.

Aim: The aim is this study is to investigate nurses and midwives' perception of their professional image and describe the factors influencing their perception.

Methods: A mixed method approach was employed. Qualitative data was first collected followed by a quantitative evaluation. Participants were recruited from six (6) regions in Ghana. Nurses and midwife age 18 years with at least one year practiced experience were eligible. Recruitment was done through oneon-one contact and via social media platforms. In depth interviews were conducted with 60 nurses and midwives and questionnaires completed by 778 respondents online. Interviews were transcribed, coded and grouped into themes and subthemes using content analysis. Mean and standard deviation were described and multi-level modelling was used to examine the personal, geographical and organizational factors influencing the contemporary image of the nurse and midwife.

Results: Nurses and midwives had positive $(M=21.31, S D=3.09)$ and negative perception of nursing and midwifery. Demographic factors such as education $F_{(3,772)}=5.10, p=.002$, professional category $F_{(2,773)}$ $=4.08, p=.017$ and rank $F_{(6,766)}=3.07, P=.002$ influenced professional image. Other qualitative determinants of image of nursing and midwifery were education and training, professionalism, clinical leadership culture and influence of regulatory body and professional associations.

Conclusion: Nursing and midwifery profession in contemporary times still carries the perspectives of the medieval age. Strategies for de-emphasizing negative factors affecting the profession needs to be explored and addressed.

\section{Background}

Nursing and Midwifery has evolved over the years into a profession seeking to sustain its autonomy. One of the key elements that will enhance the growth of nursing and midwifery is an understanding of the factors that promote or inhibit the positive image of the profession. Nursing and midwifery image is multi-dimensional, all-inclusive, and a complex concept influenced by poor communication, grooming, behaviour, gender and professional organisations [1]. Nursing and Midwifery image is viewed from the lenses of the public, media and the nurse's self-concept of professional identity. In recent times, there is an emergence of the marketing image which is characterised by advertisers using words like 'opportunity', 'rewarding' and 'travel' to attract youngsters into the nursing profession [2].

Nursing and Midwifery from its inception as a vocation, through transition into a profession and a discipline, has endured positive and negative stereotypes. The positive image considers nursing as good, 
heroic and angelic while the negative image depict nurses as sex object, naughty and handmaids [3]. The media has perpetuated branding these negative stereotypes over the years and redeeming the professional image of nursing has become a daunting task for many countries including Ghana. The interesting twist to the image debate is that while young people in developed countries are not attracted to the profession [4] developing countries like Ghana are overwhelmed with the number of young people wishing to enrol because of the opportunities [5]. The lack of interest in choosing nursing or midwifery as a career is influenced by poor working conditions and limited level of autonomy Nurses are seen as inferior to doctors and people who do not need higher education; more so the job is demanding and yet the profession is poorly understood $[6,7]$.

Multi-generation and gender differences have also influenced the image of nursing [8]. Older nurses who are expected to mentor the younger nurses have generational differences in the way they think, speak and act. These differences need to be harnessed in order to improve the professional image [8]. Male nurses and male nursing students also see nursing as a profession for men, and most of them are motivated to enter into the profession contrary to the feminist agenda that was promoted in the past [9-11]. However, some male nurses argue that the inferiority complex associated with nurses emanates from the feminist orientation of nursing. This suggest male nurses feel more confident and project a good image of nursing with their assertiveness. The lack of assertiveness is stereotyped within gender roles with accusations that nurses are unable to communicate publicly or engage the media because of the female factor [1].

Education is believed to improve nursing image since highly qualified nurses are expected to provide better quality of care [12]. This argument has been contested by critiques who suggest that education only increased the gap between theory and practice rather than enhancing the image of the profession [13]. In Ghana, nursing and midwifery education has improved, however, this is not reflecting in the improvement of the image of the profession. Previous studies on nursing image have examined the image of nursing from the perspective of the public, media, nursing students and prospective students [8]. Some studies also explored how nurses' self-concept and professional identity and the influence on image of nursing $[14,15]$.The issue of nursing image is dynamic and ought to be understood from different context. In recent times, midwives have tried to also create an identity beyond being known as nurses. It is therefore important to explore how nurses and midwives perceive the profession and the factors influencing the image of nursing and midwifery in Ghana.

\section{Methods}

\section{Study Design}

The mixed method design which involved collecting the qualitative data prior to a quantitative evaluation was employed. The phenomenon of determinants of the image of nursing and midwifery profession was explored from a pragmatic and interpretive paradigm. The qualitative component of the study employed an exploratory and interpretive approach was employed with the assumptions that nurses and midwives 
will perceive differently the image of the professions. More so, the differences and similarities of the image narratives could be thematically grouped and interpreted.

A quantitative evaluation was done subsequently to identify sociodemographic predictors of professional image. A cross-sectional survey design was used to collect data from the participants at one point in time [16-18]. Questionnaire were distributed virtually on social media platforms of nurses and midwives from January to February 2020. The specific questions to examined were: i) what is the image of nursing as perceived by nurses and midwives and ii) what are the determinants of the image from their perspective?

\section{Study setting}

Ghana has a total land area of 227,540 square kilometres with a population of 31.1 million as at 2019 (Worldometer, 2020). It is bordered by Burkina Faso in the north, Cote d'Ivoire to the west and Togo to the east. The southern boundary is covered by Gulf of Guinea, a coastline that stretches about 560 kilometres. There are ten regions namely Upper West, Upper East, Northern, Brong Ahafo, Ashanti, Western, Central, Eastern, Volta and Greater Accra regions. There are 23, 829 health facilities across the country and $56.9 \%$ of these are government owned. The total health workforce as at 2017 was 107985 with 68492 nurses and midwives. The distribution of nursing workforce shows professional nurses constituted $31 \%$, midwives $14 \%$, and auxiliary nurses $55 \%$. A total of 1241 health tutors work in health training institutions but were not classified as nurses or midwives. There are sixty-seven (67) nursing training colleges and nineteen (19) accredited universities and university colleges that offer nursing and midwifery programs. The Ghana College of Nurses and Midwives also runs specialisation programs that leads to the award of fellowship. The Nursing and Midwifery Council regulates nursing and midwifery in Ghana.

\section{Sampling Size and Sampling Technique}

Samples for the qualitative study were purposively selected through one-on-one contacts with institutional gatekeepers and sharing notice of study on social media platforms of the various regions to facilitate recruitment. Data saturation was achieved with 60 interviews.

According to data from the Nurses and Midwives Council, there are 70,996 registered nurses and midwives in Ghana. A statistical power analysis, using Gpower computer programme [19] indicates a minimum sample of 280 participants to detect medium effects ( $f 2=0.15)$ with $95 \%$ power and 0.05 alpha. The convenient sampling technique was used in collecting data since recruitment was based online completion of questionnaire by respondent. Nurses and midwives were recruited using multiple portals, including social media platform, namely Facebook and WhatsApp and poster recruitment.

\section{Data collection tool}

A Semi structured interview guide and questionnaire were used to collect data. The semi-structured interview guide was used to conduct the in-depth interviews. The main questions were: What is your 
assessment of the image of nursing and midwifery? What factors in your view, affect the image of nursing and midwifery? Probing questions were asked to explore the influence of education, leadership, regulation and other institutional factors on image. The nature of probing questions varied relative to the responses of participants.

The questionnaire was developed based on the results of the qualitative data generated as part of the study, taking into consideration the extent literature on the subject. Since we were interested in ensuring ecologically valid responses that accommodate the cultural specificities and nuances of Ghana, basing the questionnaire development on the results of the qualitative study largely ensured that this objective was achieved. The questionnaire was prepared online using google form.

Thematic areas and constructs derived from the qualitative exploration of transition and innovation was used to construct a 5 to 7 level Likert scale with four domains Image of nursing and midwifery 5 items

Additionally, information about sociodemographic characteristics of respondents were included and used as covariates in modelling for association and predictors of transition in nursing and midwifery. The questionnaire which was electronic, had an introductory part that summarizes the study purpose, benefit and risk. Voluntary participation and right of withdrawal were emphasized.(Refer Supplementary File)

\section{Data collection method}

Data collection involve use of interviews and virtual completion of questionnaires. The in-depth individual face-to-face interviews were conducted using interview guide. Audio recorded interviews were conducted mainly in English with nurses and midwives. Interviews were conducted by five (5) co investigators who were distributed across the six regions. A minimum of one (1) week was spent in each region in conducting the interviews. Information was obtained on participants' personal characteristics, their perception of the image of nursing and midwifery in Ghana. Each interview lasted for a minimum of 45 minutes. Transcripts were created for each interview and code names were assigned to ensure anonymity. Participants right to inclusion and withdrawal from study were protected. Field diaries were used to capture events and observations that enhanced the interpretation of the data.

The questionnaire link was also sent via private social media inboxes of randomly selected list of contact in WhatsApp as a broadcast. Reminders were sent one week when feedback was not received. The questionnaire had screening questions such as age, status (i.e., student versus practitioner) duration of practice to ensure that only those meeting the eligibility criteria noted previously participate in the study. In order not to exclude nurses and midwives who have no access to digital platforms, paper-based questionnaire was made available to such individuals but none requested for paper completion

\section{Data analyses}

Qualitative and quantitative data were collected and analysed separately. Content analysis which involved extracting codes, categorising and grouping themes and subthemes based on the pattern of narratives was done. Transcripts were read severally and coded by four team members. The team 
reviewed the codes and categories to make decisions regarding revision or deletion. Themes and subthemes were developed and described with reference to field data, memos and direct quotations from the interview.

IBM SPSS) version 23.0 was used organise data for descriptive analyses (mean, standard deviation, and frequencies) and inferential statistics. A multi-level modelling was used to examine the influence of personal, geographical and organizational factors influencing the contemporary image of the nurse or midwife was used. Data exploratory techniques, namely principal component analysis was conducted to determine the utility of the study items, whereas reliability of the study measures was examined using Cronbach's Alpha coefficient.

\section{Ethical issues}

Ethics approval was received from the Ghana heath service (Protocol No. GHS-ERC/011/05/19). The right to privacy, anonymity and confidentiality were protected throughout the study. Interviews were schedule and venue determined with participants. Permission was sought for audio recording and all personal identifiers excluded from the transcript. Prior to sending questionnaire to individuals, desensitization and information were provided to respondents in a debriefing session to discuss the purpose of the study with prospective respondents via the social media link. The information sheet was shared to others on regional nursing and midwifery social media platforms for interested respondent to read and ask questions. The structure of the link shared included the collection of email and contacts of respondents. Security feature was integrated to minimize data fraud and protect respondents from invasion of privacy. An appreciation note was added after completing questionnaire as a psychological compensation.

Ethical issues relating to cyber security were anticipated for reference to the cyber security team of the University of Ghana Computing Systems for advice however, none was observed. This is a commissioned study funded by the Nursing and Midwifery Council of Ghana, however, there is no conflict of interest situation.

\section{Findings}

\section{Assessment of image of Nursing and Midwifery}

Participant accounts and quantitative evaluation suggest the image of nursing and midwifery was both good and bad.

\section{Positive image of nursing and midwifery}

Nurses and midwives conduct during care were used as basis for assessing the professional image. A quantitative evaluation of the image of nursing and midwifery showed that respondents rating the image of nursing a five-point Likert scale was positive $(M=21.31, S D=3.09)$. Measures assessed included intelligence, importance, independence, respect for profession and self-concept. Results as shown in 
table 1 revealed that nurses and midwives had a positive image about themselves and the profession. The importance of nursing and midwifery had the highest score $M=4.7, S D=.902$ ).

\section{Table 1 assessment of image}

\begin{tabular}{|lcc|}
\hline Items & M & SD \\
\hline It takes intelligence to be a nurse/midwife & 4.43 & .911 \\
\hline The services given by nurses/midwives is as important as that given by physicians & 4.65 & .902 \\
\hline Nurses/midwives are capable of independent practice & 4.29 & 1.01 \\
\hline Nursing/midwifery is a respected profession & 4.04 & 1.10 \\
\hline Nurses/midwives feel good about what they do & 3.90 & 1.02 \\
\hline Image score & 21.31 & 3.09 \\
\hline
\end{tabular}

The narrative on the perception of nursing and midwifery suggest a conditional positive outlook.

"...for me, if you look at their work in general, some are good and some are not and depends on the entry point (NER GNE1).

\section{Negative image of Nursing and Midwifery profession}

Results in table 1 indicates lower score $(M=3.9, S D=1.02)$ for whether nurses and midwives feel good about what they do. This reflects the negative stories about perception of image. Some participants compared events of the past and noted a declining image of the nursing profession in particular. A participant noted the influence of the negative image on the willingness to publicly identify yourself as a nurse or midwife.

"In fact, it is so damaging that wearing uniforms in public is not something that most of us would like to do and even sometimes in places you do not want them to identify that you're a nurse or a midwife. Because the utterances that you hear you just have to hide back for as much as you can until you leave the place." (NR RMC)

The negative perception of the image of nursing was observed to be influenced by five factors which were summarised in figure 1 and discussed in subsequent sessions

\section{Determinants of nursing and midwifery image}

The key determinants were demographic characteristics, education and training, professionalism, clinical leadership culture and support/regulation of practice as illustrated in figure 1. Demographic factors influencing image of nursing were derived through quantitative analysis while the remaining factors emerged from qualitative outcomes. A parallel approach to reporting was therefore employed. 
Demographic factor influencing assessment of the image of nursing and midwifery profession

Education, professional category, rank and duration of practice influenced the image of nursing and midwifery as shown in table 2. Educational background demonstrated statistically significant effect on nursing image, $\left.F_{(3,772)}=5.10, p=.002\right)$. Master's degree holders rated nursing/midwifery image high, compared with holders of diploma $(p=.004)$. The relationship between how long the participants have work and image of was statistically significant suggesting that individuals who have practiced longer are more likely to have positive image of nursing.

Table 2 Demographic factors influencing image 


\begin{tabular}{|c|c|c|c|c|}
\hline \multirow[t]{2}{*}{ Variable } & \multicolumn{3}{|c|}{ Descriptive } & \multirow{2}{*}{$\begin{array}{l}\text { Inferential } \\
\text { F-test values }\end{array}$} \\
\hline & $\mathrm{n}$ & M & SD & \\
\hline Gender+++ & & & & $.20, \mathrm{~ns}$ \\
\hline Male & 239 & 21.36 & 2.70 & \\
\hline Female & 539 & 21.32 & 3.17 & \\
\hline Profession Category & & & & $4.08 *$ \\
\hline Nurse & 592 & 21.22 & 3.00 & \\
\hline Midwife & 82 & 21.23 & 2.86 & \\
\hline Nurse-Midwife & 102 & 22.14 & 3.27 & \\
\hline Education & & & & $5.10 \star \star$ \\
\hline Diploma & 175 & 20.80 & 3.02 & \\
\hline Undergraduate & 370 & 21.25 & 2.98 & \\
\hline Masters & 221 & 21.83 & 3.09 & \\
\hline $\mathrm{PhD}$ & 10 & 23.10 & 2.02 & \\
\hline Place of Work & & & & $1.86, \mathrm{~ns}$ \\
\hline Training School & 85 & 21.84 & 3.17 & \\
\hline Teaching hospital & 155 & 21.15 & 2.70 & \\
\hline GHS Facility & 312 & 21.43 & 2.83 & \\
\hline CHAG Facility & 112 & 20.65 & 3.92 & \\
\hline Private hospital & 39 & 22.21 & 1.79 & \\
\hline Others & 76 & 21.34 & 1.70 & \\
\hline Place of Training & & & & $1.96, \mathrm{~ns}$ \\
\hline Public University & 131 & 21.35 & 3.29 & \\
\hline Private University & 43 & 21.35 & 3.59 & \\
\hline Public NTC & 557 & 21.24 & 2.96 & \\
\hline Private NTC & 13 & 21.15 & 2.88 & \\
\hline Quasi-Public & 38 & 22.66 & 2.27 & \\
\hline Professional Ranks & & & & $3.07^{\star \star}$ \\
\hline Staff Nurse & 70 & 20.16 & 2.98 & \\
\hline
\end{tabular}




\begin{tabular}{|llll|}
\hline Senior Staff & 105 & 21.29 & 2.59 \\
\hline Nursing Officer & 170 & 21.35 & 2.91 \\
\hline Senior Nursing Officer & 189 & 21.30 & 2.95 \\
\hline Principal Nursing Officer & 98 & 21.96 & 2.65 \\
\hline DDNS \& above & 84 & 21.90 & 4.12 \\
\hline Others & 57 & 21.44 & 2.33 \\
\hline
\end{tabular}

Professional category exhibited statistically significant effect on nursing image, $F_{(2,773)}=4.08, p=.017$. Post-hoc analysis with Bonferroni procedure revealed that Nurse-Midwife reported significantly better image of nursing/midwifery than Nurses $(p=.014)$ but no difference with midwives $(p=.128)$.

Professional rank exhibited statistically significant effect on image of nursing/midwifery, $F_{(6,766)}=3.07$, $P=.002$, with staff nurse more likely to report low image of nursing/midwifery than principal nursing officer $(p=.002)$ and individuals with qualifications from DDNS and above $(p=.006)$.

There is no statistically significant relationship between age and image of nursing as reported by the participants. The probable reason could be that the although the age range is from 23 to 64 , about $75 \%$ were 39 years and below. This age restriction could reduce the variance needed for statistical significance. Gender, place of work and place of training did not significantly influence participants rating of nursing /midwifery image.

\section{Influence of Education and Training on professional image}

The nature and quality of educating nurses and midwives was recognised as a factor influencing the professional image. This was manifested through pre-professional motives for choosing nursing and midwifery, increases in student enrolment and competency of tutors.

\section{Pre-professional motives: Intrinsic and extrinsic motivation}

The image of nursing and midwifery was perceived to be affected by intrinsic and extrinsic motivation. This was reflected in the dedication and commitment to positively enhance the image of the profession. Additionally, the choice of nursing and midwifery because of easy employment and travel opportunities served as a pull factor that attracted different people into the profession. Some regarded nursing as a gateway to career development while others were coerced by significant others to pursue nursing or midwifery.

Choosing nursing and midwifery because of the passion to care and serve was obvious in the work of those who did nursing out of free will. A participant comparing practice experience said: 
"... The work was efficient as compared to now." "We were more dedicated, more committed and are able to handle the patient very well... (UER_NS2)

However, participants noted choice of nursing and midwifery based on alternative reasons was common. A participant opined that:

"It was easy to get employment so you know, people felt that, instead of going into one profession or the other, they could find themselves in nursing." (ER GRNMA)

Aside early employment, others indicated that entering into nursing was a conduit for higher achievement and financial gains. The perception of nursing as a job for earning income in some cases is affected quality of care as reflected in the following:

They want to just get a job but do not have the heart. If you don't have the heart for the job, it means you cannot do it well. (UER GNE1)

Nursing was also seen as the gateway for international travel. The perception that visa acquisition was easier for nurses coupled with shortage of staff in some developed countries fuelled the travelling agenda. A participant commented that:

"I would say that beginning from the early part of 2000, people realized that nursing was the profession that if you decide to enter you could easily travel outside." (ER GRNMA)

Additionally, coercion from significant others for people to select nursing and midwifery as a career is blamed for the quality of students enrolled.

"It's a generational thing, because sometimes they will tell you that me, I didn't like nursing but I was forced into it. So, it is a generational thing starting from the politicians. They are poking their nose so much into the profession" (GAR GHS)

Individual selecting nursing to satisfy parental or political influence turn to have poor work culture and this is perceived to be affecting the image of the nursing and midwifery profession.

\section{Increase in Student enrolment}

Participants reported that increase in student enrolment affected the quality of training of nurses and midwives in Ghana. The quality of teaching, class control and supervision of students has been grossly compromised.

"... we are also hit by huge numbers, so what type of quality teaching are you going to do? What supervision are you going to do? So eventually they come out and they cannot perform. You are going to have just a handful who are interested in the job who can do something but the rest cannot. So, you see them, they have nothing to offer..." (UER GNE 1) 
The increase numbers were believed to result in lack of attention, poor clinical exposure and inadequate supervision.

\section{Experience of Tutors}

Experience of tutors before recruitment to teach in the nursing and midwifery training colleges was considered to affect the training of nurses and midwives. Tutors with more clinical practice were preferred to younger ones who may not have the needed clinical skills. Participants opined that some tutors are 'half-baked nurses', immature and unable to train others.

...the tutors are too young, is like the nursing training college is open up too much... if you talk they say you are'collo' [out of date]. Those days you can't teach here if you have not practiced for some number of years... the immaturity is too much in the schools... now whether you are a nurse or not you can teach here so everybody comes (NR RME)

The concerns about the competency of trainers of nurses and midwives included the use of avoidance by some tutors to cover up for their inability to give appropriate feedback and support students' learning. Experienced and competent tutors will train nurses with the right skills and attitudes which will promote positive image of the profession.

\section{Contribution of Professionalism to Perception of image of Nursing and Midwifery}

Professionalism of the nurse and midwife in Ghana was described in relation to the physical appearance, competency, attitude and behaviour. According to participants, the image of nurses and midwives in the public eye comes with mixed reactions. The public perception is both positive and negative. However, participant noted a dwindling image attributable to public perception and negative media reports. A participant said:

"...those who know will say that our image is going down. Yes is about $65 \%$ and the no is about $35 \%$ because when you open the radio and they are talking about Nurses everybody lambast us, everybody keep on saying that we are not up to the task" (WR PHNE)

\section{The physical appearance of Nurses and Midwives}

The physical appearance of the nurse or midwife was a quick and easy lens through which participants viewed professionalism. All participant admitted that nurses and midwives are unique professionals who were easily identified by their attire. However, it was reported that most nurses in recent times were either dressed in plain clothes or dressed incompletely. This makes differentiation between a nurse and patient difficult and therefore lowered the confidence and trust that patients had for the nursing and midwifery profession.

"...some of us come to work in the morning dressed anyhow and at times we can't even differentiate between a nurse and a patient. Very often, the client is in mufti and the nurse is in T-shirt and jeans, so 
who is attending to who and how do you expect this patient to have that confidence and trust in you?" (AR RHD)

It was also reported that nurses especially are perceived in a more negative professional outlook than midwives. Typically, female nurses were likened to prostitutes because of the way and manner they dressed. Participants mentioned that the younger nurses dressed in short and tight uniforms and use make-ups on their faces as though they were prostitutes. As a result, they do not attract the patients they were to serve.

... some even associate female Nurses to prostitutes, yeah you talk about female Nurses and that is the next word that you hear from the patient, because the dressing alone, you see the dress being short very tight and I wonder whether we are here to work or to showcase our body" (WR NS2)

Similarly, a few participants speculated about the absence of a specific type and style of uniform for nurses makes it difficult for differentiation. Consequently, negative attributes of other individuals assumed to be nurses or midwives affects the image of the profession.

"... the media most of the time are unable to differentiate between Registered Nurses and Health Assistant. ...Probably it was a cleaner or it was a health assistant; that differentiation is not there" (GAR $\mathrm{VVU})$

The colour of uniform was perceived to affect how the public differentiated qualified nurses from the other professionals Community Health Nurses noted they were perceived as 'village nurses' who were not real or qualified.

\section{Behaviour and attitude of the nurse and midwife}

According to some participants, some nurses demonstrated caring attitude however, task shifting and use of mobile phones contributed to negative attitude. Task shifting was prevalent among nurses and midwives working during night shifts. Some of them usually sleep resulting in neglect of patients or assigning of roles to patient relatives. In some instances, patients or their relatives had to discontinue infusion and transfusion lines when the nurse were out of reach.

"In fact, the truth of the matter is that during night shifts, most of our activities are given to the patients to do. The relatives see that what we [nurses] do for the patient, they can also do. So, to them, nursing is nothing special" (NR GNC)

The use of mobile phone and social media browsing during working hours was considered inappropriate leading to poor attitude towards care. Some participants noted:

Some nurses especially the young ones are usually on their phones. Sometime when you question them, they will say "my mother has called" (AR RMC 1) 
Nevertheless, a few participants acknowledged the positive use of mobile phones and internet during nursing clinical hours.

\section{Clinical competency}

Nurses and midwives with expertise and control over their clinical skills were considered to project a good image of the profession. Similarly, challenges associated with translating theory into practice affected professional image. The incompetency resulted in poor attitude towards work. Most of the participants attributed this to lack of mentorship or the lack of resources to support care.

"I think there is a gap between what is learnt and what is practiced, thus, one has to do with practical and then experience actually doing the work practically because when you come out you may have the knowledge but you may not have the practical experience. How to also apply this knowledge becomes a challenge between those in the wards and those who have just come from school" (WR RMN).

Some participants reported that nurses desire for higher academic qualifications without focusing on the skills acquisition. Consequently, the increase in the number of highly qualified professionals is not reflecting on the standard of care.

"... I was thinking that with the universities springing up doing a lot of Degree courses, Mphil, BA Igraduate and undergraduate program] we have a whole lot of courses being done but then it is not really impacting on our practice" (WR NS2)

Concerns were raised regarding competency in meticulous documentation. According to a participants, nurses and midwives in Ghana may not be able to redeem their images without proper documentation processes.

Documentation has been a very big problem. I went out with the council here so for supportive supervision in 3 health facilities. It was hell. Documentation is just a problem. How to chart vital signs is a problem and yet a lone to talk about the patograph. (UER NS1)

\section{Influence of Clinical Leadership Culture on professional image}

Clinical leadership culture was described with regards to mentorship, monitoring and supervision, resource constraints, educational advancement opportunities and staff

motivation. The adequacy of clinical leadership promoted positive professional image while inadequacy of same projected a negative image.

\section{Mentorship}

It was palpable from the data that the young cohorts of nurses/midwives were not ready to learn from the old experienced nurses/midwives or that the old experienced nurses/midwives were not supportive enough to the new and novice nurses and midwives. 
"The reason is that the young ones are not ready to be taught. Yes they don't make themselves available. They have their own agenda" (GAR DNS)

"May be some of us, the old ones, might not have the knowledge, or they are not ready to also share their knowledge with the young ones." (NR PHNC1)

The results presented evidence that participants especially the older generation of nurses/midwives including patient relatives preferred to be cared for by retired nurses or old nurses instead of the calibre of young generation of nurses. This preference was a consequence of the ill-preparedness in terms of clinical skill and attitude on the part of the young crop of nurses.

"The experienced nurses, some of them are okay, they're trying to mentor others, and others too are of the view that, well, these young people, they don't respect, so I'll not even mind them" (UER RME)

On another breath, it was revealed that among the old experienced nurses are some who were willing to mentor and groom the young nurses only if they showed seriousness to learn.

\section{Monitoring and Supervision}

Occasional snap supervisory visits were recognised as a mean of ensuring adherence to standard practice which promote positive professional image. Participants generally argued that clinical leadership culture suggests inadequate supervision and monitoring which has accounted for the low image of nursing and midwifery profession. Commenting of relevance of supervisory visit from Nursing and Midwifery Council, a participant noted that:

"... once in a while I think they [regulatory body] come around but I think they have regional offices so I think they can empower them with that mandate so that even if it once every 6 months, they can go around the schools, they'll listen to some of the things on the ground" (UER RME)

Participants' acknowledged the presence of supervisors but preferred a decentralisation of supervisory roles to regional offices of the Nursing and Midwifery Council. Regular supervision in training schools were also recommended. Another participant reiterating the value of supervision bemoaned the challenges associated with effective supervision.

"We have trained them and we have to supervise them to make sure they are putting up the requisite behaviour. However, this is a major challenge. The supervision is a challenge so we have numerous reasons for this but all is not lost." (GAR DNS)

Strengthening monitoring and supervision is expected to ensure that nurses and midwives have the requisite qualification and skills to efficiently provide care. Additionally, supervision will enhance appropriate behaviour consistent with professional standards.

\section{Resource Constraints}


Resources availability promotes work efficiency while resource constraints affects work output. The poor image of nursing image was attributed to resource constraints. Resources such as personnel and equipment facilitate the quality of care. The absence of basic work tools for nurses and midwives result in frustration, disappointment and loss of hope for nurses and their clients.

"equipment are the main challenge nurses do face ... most of the times we have to get a gadget to work with, and then because is not available we have to refer the clients. I think the referral system too is a bit poor "(WR NS1)

The findings showed that there were times patients were referred to other facilities because of limited resources. And even before the referral, the basic intervention the nurse would have done was not carried out because of resource constraints including lack of beds, oxygen and consumables.

Resource constraints affected timely implementation knowledge and skills acquired as part of continuous education programs. Providing nursing care with limited resources attributed to lack of funds or time directly affects quality of care and indirectly influence how nurses and midwives are perceived by their clients.

\section{Educational Advancement Opportunity}

Nursing and midwifery made strides over the years in the introduction of advanced courses of higher learning and opportunities to obtain career development. This was noted among participants as a positive projection of image of the profession.

“...I think right now Nurses we are aiming higher trying to go more into research, trying to develop our own body of knowledge. I think right now Nurses and midwives trying now compared to those times." (WR NS2)

However, commitment to the work remained problematic among the young generation of nurses/midwives.

"They advance and they learn more things which help the patient management. So, these days the young ones they advance in their knowledge than we the first people. ...they have the skills but it's only that some of them the commitment is not there" (UER NS2)

The findings showed reduced enthusiasm among the old experienced nurses to read books or upgrade themselves with current knowledge in nursing and midwifery.

\section{Motivation to Staff}

Motivation to staff reflected as a challenge for nurses/midwives at the work setting. Participants lamented about the demotivating conditions of work. They indicated the type of motivation they needed in order to be happy at work. They desired health insurance cover, fiscal remuneration and fair distribution of accommodation facilities. 
"I am getting only my salary there is no motivation, there is no incentive and then when you ask your colleagues who are in different institutions they will say oh Charlie the hospital is trying to give us some coins and we can use it to solve certain problem, and you think about that and you think that oh you are being neglected." (NR NS2)

Taking part in decision making was one aspect noted to make people motivated. It was evidenced that, intrinsically, nurses feel motivated and committed to the implementation of ideas or strategies that they were part of during the decision-making process.

"... I will be motivated to do something once I get to know that I am part of it in originating the idea ... and we have all pledged our commitment, we will not want it to fail." (UER GNC)

Intrinsic motivation was noticed to be the preferred style of motivating staff by management. Nurses grumbled that management most at time are reproachful to extrinsic motivation when raised at any given opportunity.

Other examples of motivation expressed by nurses included the distribution of uniforms, the institution of extra work hours rewards and the avoidance of delays in promotions when they are due.

\section{Influence of Support and Regulation: Professional Associations and the Regulatory Body}

Participants opined that actions and inactions of the regulatory body which is the Nursing and Midwifery Council of Ghana and professional associations such as Ghana Registered Nurses and Midwives Association contributed to the image of the profession. The was a perception of lack of support and weak control of these bodies which accounted for the negative perception held among the participants.

\section{Professional association support}

Support from professional associations was considered to be important factor in promoting the image of the nursing and midwifery. However, participants generally were oblivious of the contribution of the Ghana Registered Nurses and Midwives' Association in the functions of nurses and midwives in Ghana. The association was only known for their monthly financial deductions from members account. It was a general believe that when nurses get into trouble, they are left to their fate. The associations are not seen as fighting for the interest of the individual members.

I don't see them fighting the nursing course. If they are doing it then they are not meeting our needs. We are told they are for bargaining, that is not the issue I am looking but you have instances where nurses get into serious trouble or have issues and they are left on their own..." (UER GNE2)

\section{Enforcement of rules, regulations and sanctions}

The participants identified the professional associations and the Nurses and Midwifery Council as two bodies which can regulate activities of nurses and midwives to redeem the professional image. Participants suggested a host of sanctions and other punitive measures to be enforced. 
"...everybody has to be on board nurse managers, the training institutions, the policy makers, the institutions, the clergy both Muslims and Christians and families. We all need to instil values in the students. If they come to the schools the teachers have a part to play" (GAR GHS).

The interview session should be rigorous to actually sieve those who have passion for nursing from those who do not have. Further, careful scrutiny through preliminary examinations should be brought back so that students would justify their inclusion and continuation of the nursing course after one year of their admission.

"...I think it will start from the interview when we are really taking new Nurses at the various institutions that is where we should really make sure that the person love the job. ...It is not always about grade, no this person passed very well so we should admit the person, you have to really look at the person's attitude, the love for the job "(WR NS2)

Upgrading oneself through workshops and other specialty programmes were noted important to keep abreast with nursing knowledge.

"Nursing really needs reshaping and rebranding. ... we can do this by empowering ourselves through workshops, through going for further studies specially with the specialties, GCNM (Ghana College of Nurses and Midwives) has come, GCNM is really trying products from GNCM you can see they are totally different from the post basic now the degree...",(NR NS1)

It was indicated of the importance of ensuring a credible licensing examination. Participants expressed that the Nursing and Midwifery Council should always maintain examination standards, visit facilities and observe nursing practice. By this, it would improve practice once shortcomings are identified and solutions proffered.

"So council, in order to maintain standards and what not, they should relook at the way their exam is conducted and take charge, financial charge of their exams and also make sure they regularly visit facilities, observe nursing practice, and if there are shortcomings they should be able to provide solutions" (UER GNC)

\section{Examination standards}

The examination standards of NMC was reported to have fallen. During examinations, student contribute monies and care for the examiners with gifts and many other things. This was reported to be compromising the examination process because that puts the examiners in tight situations and becomes very difficult to turn and fail students even if they did not perform well.

"What I have observed is that sometimes you have these students, who will have to, sometimes put together some small tokens, as gift. So obviously, ... why will you stand before that same person and fail the person? You know, it won't be fair and I think that is something Council should be looking at" (UER $\mathrm{GNC})$ 
The results showed that council should step in and determine the number of students that is admitted since they have a regulatory oversight responsibility. This was believed to contribute to the 'whole sale' production of nurses/midwives.

"...though it was not NMC (Nursing and Midwifery Council of Ghana) that admitted them [students] but I think they play a role as a regulatory oversight body, they can determine how many school have the manpower and facilities and insist on the number they can train properly because it is proper training that will determine the kind of nurses we are producing.." (UER GNE2)

\section{Discussion}

The aim of this study was to assess the image of nursing and midwifery and determine the factors influencing the image of nursing and midwifery profession in Ghana. The findings suggest that nurses have positive image of themselves but a negative image of the profession. The perception of image was influenced by education and training, professionalism, leadership culture, regulation and demographic characteristics. The positive image was reflected in their assessment of their self-concept which revealed high rating for intelligence, importance of services, independent practice, respect and a sense of good feeling. Karanikola et al. (2018) noted the perception of nurses about the image of the profession is an interplay between self and professional concept which is manifested in role performance. The quantitative evaluation revealed that nurses and midwives with higher education and rank, longer duration of work experience were more likely to have a positive image of themselves and the profession. These characteristics and others such as tradition, cultural and social values have been observed to influence public and self-created image of nursing [12].

Education and training are perceived to have a ripple effect on professional image of nursing and midwifery. Pre-professional motives, increase in enrolment of students and the competency of tutors affects the quality of nurses and midwives graduating from the various institutions. Nurse and midwives enrolling into nursing by choice based on calling and passion promoted positive perception of the profession. Some studies have suggested voluntary selection of nursing as career, enhances self-concept and professional valuing among nurses, nursing students and prospective students [20]. However, extrinsic drives such as employability, travel opportunities and family influence were perceived as minimising professional commitment. Nursing students had a positive attitude towards the profession in spite of family influence in other reports. Notably the desire to travel positively influenced were observed to influence attitude [5]. The findings in [5] does not support the fears expressed about professional image being influenced by job and travel opportunities in the current study. Nevertheless, the differences observed could be explained in terms of the differences in the participants in this study.

Another factor influencing training of nurses and its impact on nursing image is the enrolment numbers leading to increase in class size. There is paucity of data on class size and faculty-student ratio but Bell, Rominski, Bam, Donkor and Lori (2013) reported a ratio of 1:76 lecture to student for baccalaureate programs. Observation and personal experiences suggest the numbers far exceed the projection as most 
of the training colleges have class sizes ranges between 100 and 500 students. The resources available for training are also inadequate as reported in this study. Measures were implemented to bridge the faculty gap by enrolling younger nurses and midwives with minimal clinical practice experience [21]. The acceleration in recruiting younger nursing faculty fuelled the perception of nursing tutors lacking clinical skills thereby unable to meet expectations. Participants opined that the poor image of the nursing profession has been affected by the employment of tutors with minimal practice experience who themselves need to be mentored. The phenomenon of younger nurses and midwives in training institutions depict the consequences of the changes in the sociodemographic dynamics in nursing and midwifery in Ghana generally [22]. Continuous quality education and training among clinical nurses and tutors is essential. This will create opportunities that strengthen core professional values, improves practice and increase competency gaps $[8,15]$.

Physical appearance, behaviour and clinical competence affects how nurses and midwives discharge their duties. The actions and omissions occurring in the performance of professional responsibilities was mentioned as a factor influencing the image portrayed in the current study. In this regard, nurses and midwives dressing inappropriately was noted as contributing to the negative image of the profession. Wills, Wilson, Woodcock, Abraham, \& Gillum, (2018) noted appearance of the nurse and midwife is considered a trademark of professionalism which shows in the caring attitude, the way the nurse dresses in uniform, the smiles and friendliness. It should be easy to approach and interact freely with the nurse who is also trustworthy. These transcending values reflects the angelic image that the public and media expects $[7,20]$. Nurses who are competent are perceived to positively influence the image of nursing as they provide safe and comfortable care. Nurses who are rude and insensitive to patient's needs contribute to propagating the negative media labels and public perception of the profession [24].

Mentoring, monitoring, supervision, resource availability, educational advancement opportunities and staff motivation were also observed as leadership culture factors affecting the image of nursing and midwifery. Firm and fair leaders serve as role models in guiding the younger generation to adopt the right attitude to work through mentoring [22]. There is evidence that support improvement in care, job satisfaction and retention on nurses due to adequate motivation including opportunities for higher education [7]. Structured mentoring processes which is not available in our current study setting needs to be engineered to ensure nurses and midwives represent a positive image of the profession.

Support from professional associations, enforcement of standards and sanctions and improving examination processes were mentioned as factors that can contribute to improving the image of the nursing and midwifery. Rezaei-Adaryani et al., (2012) argued that the power of nursing as professional organizations could drive the image of nursing because of the numerical strength. However, fragmentation and the lack of unity of purpose will affect progress. Nurses and midwives need to be politically conscious and collaborate effectively to build the image of the profession.

\section{Conclusion}


The image of nursing and midwifery profession in contemporary times still carries the perspectives of the medieval age. Nurses and midwives who are expected to have very positive image of the profession still have reservations about the quality of training, professionalism, clinical leadership and the regulatory regimes. Building strong and resilient training institutions that ensures recruitment of experienced tutors, streamlining the number of students enrolled is necessary. Mentoring roles needs to be formalised and structures to enhance clinical leader. Regulators should enforce standards guiding professional behaviour and appropriate sanctions without political interference. Appointing image ambassadors who will assess and respond promptly to issues affecting the image of nursing and midwifery is worth exploring.

Further studies that employ interventions for improving the positive factors is recommended. In spite of employing a pragmatic paradigm in exploring the phenomenon in this study, the qualitative outcomes were not extensively validated. There are no tools for assessing the various domains (education, professionalism, clinical leadership culture and regulatory support). Others studies could target a more extensive evaluation of the influence of these factors which are seemingly contextual.

\section{Declarations}

\section{Ethics approval and consent to participate}

Ethics approval was granted by the Ghana Health Service Ethics review committee and all participants signed the informed consent form.

\section{Consent for Publication}

Not applicable in this instance

\section{Availability of data and material}

All data generated or analyzed during this study are included in this article and its supplementary information files

\section{Competing interests}

The authors declare no competing interest in the conduct of this study

\section{Funding}

This research has received partial funding from the Nursing and Midwifery Council of Ghana to support only data contribution.

\section{Authors' Contributions}

GD contributed to conception, design, interpretation and drafting of manuscript 
JK contributed to conception, design, interpretation and review and is the corresponding author. LAO, AKA, CAA, SA contributed to conception, design, interpretation and review

IA, PW and FN contributed to conception of project and review of manuscript. LA the principal investigator, contributed to conception, design, interpretation and review of manuscript. All authors have approved the manuscript and accountable for their contributions.

\section{Acknowledgements}

The authors acknowledge Maria Iddrisu, Moses Tia and Yakubu Hamidu Nabila who assisted with coding the data. We acknowledge directors of nursing and midwifery in the selected institutions for their role in recruitment of participants.

\section{References}

1. Rezaei-Adaryani M, Salsali M, Mohammadi E. Nursing image: An evolutionary concept analysis. Contemp Nurse. 2012;43(1):81-9.

2. Tuckett A, Kim H, Huh J. Image and message: Recruiting the right nurses for the profession. A qualitative study. Nurse Educ Today. 2017;

3. Price SL, Mcgillis Hall L. The history of nurse imagery and the implications for recruitment: A discussion paper. J Adv Nurs. 2014;70(7):1502-9.

4. Zarea K, Negarandeh R, Dehghan-Nayeri N, Rezaei-Adaryani M. Nursing staff shortages and job satisfaction in Iran: Issues and challenges. Nurs Heal Sci. 2009;11(3):326-31.

5. Mwini-Nyaledzigbor PP. Psychosocial Factors Influencing the Perception and Choice of Nursing as a Profession: A Study at Korle - Bu Nurses' Training College. Psychol Behav Sci. 2014;3(1):25.

6. Girvin J, Jackson D, Hutchinson M. Contemporary public perceptions of nursing: a systematic review and narrative synthesis of the international research evidence. J Nurs Manag. 2016;24(8):994-1006.

7. Glerean N, Hupli M, Talman K, Haavisto E. Young peoples' perceptions of the nursing profession: An integrative review. Nurse Educ Today [Internet]. 2017;57:95-102. Available from: http://dx.doi.org/10.1016/j.nedt.2017.07.008

8. Gunawan J, Aungsuroch Y, Sukarna A, Nazliansyah, Efendi F. The image of nursing as perceived by nurses: A phenomenological study. Nurs Midwifery Stud. 2018;

9. Christensen $\mathrm{M}$, Welch $\mathrm{A}$, Barr J. Nursing is for men: a descriptive phenomenological study. Contemp Nurse [Internet]. 2018;54(6):547-60. Available from: https://doi.org/10.1080/10376178.2018.1512876

10. Juliff D, Russell K, Bulsara C. The essence of helping: significant others and nurses in action draw men into nursing. Contemp Nurse [Internet]. 2017;53(2):156-66. Available from: http://dx.doi.org/10.1080/10376178.2016.1254567 
11. Yi M, Keogh B. What motivates men to choose nursing as a profession? A systematic review of qualitative studies. Contemp Nurse. 2016;52(1):95-105.

12. Hoeve $Y$ ten, Jansen $G$, Roodbol P. The nursing profession: Public image, self-concept and professional identity. A discussion paper. J Adv Nurs. 2014;

13. Moon JA. Reflection in learning and professional development: Theory and practice. Routledge; 2013.

14. Karanikola M, Doulougeri K, Koutrouba A, Giannakopoulou M, Papathanassoglou EDE. A phenomenological investigation of the interplay among professional worth appraisal, self-esteem and self-perception in nurses: The revelation of an internal and external criteria system. Front Psychol. 2018;9(OCT):1-12.

15. Çöplü M, Tekinsoy Kartın P. Professional self-concept and professional values of senior students of the nursing department. Nurs Ethics. 2019;26(5):1387-97.

16. Creswell JW. A concise introduction to mixed methods research. SAGE publications; 2014.

17. Polit DF, Beck CT. Essentials of nursing research: Appraising evidence fornursing practice. ed Wolters Kluwer/Lippincott/Williams Wilkins Heal Philadelphia, PA, USA. 2014;

18. Grove SK, Gray JR, Burns N. Understanding Nursing Research Buliding an Evidence-Based Research. St Louis, MI Elsevier Saunders Rachel Pless, Periann Rogers. 2015;

19. Faul F, Erdfelder E, Lang A-G, Buchner A. G* Power 3: A flexible statistical power analysis program for the social, behavioral, and biomedical sciences. Behav Res Methods. 2007;39(2):175-91.

20. Price SL, Mcgillis Hall L, Angus JE, Peter E. Choosing nursing as a career: A narrative analysis of millennial nurses' career choice of virtue. Nurs Inq. 2013;20(4):305-16.

21. Bell SA, Rominski S, Bam V, Donkor E, Lori J. Analysis of nursing education in Ghana: Priorities for scaling-up the nursing workforce. Nurs Heal Sci. 2013;15(2):244-9.

22. Asamani JA, Amertil NP, Ismaila H, Francis AA, Chebere MM, Nabyonga-Orem J. Nurses and midwives demographic shift in Ghana - The policy implications of a looming crisis. Hum Resour Health. 2019;17(1):1-5.

23. Wills NL, Wilson B, Woodcock EB, Abraham SP, Gillum DR. Appearance of Nurses and Perceived Professionalism. Int J Stud Nurs. 2018;3(3):30.

24. Hallam J. Nursing the image: media, culture and professional identity. Routledge; 2012.

\section{Figures}




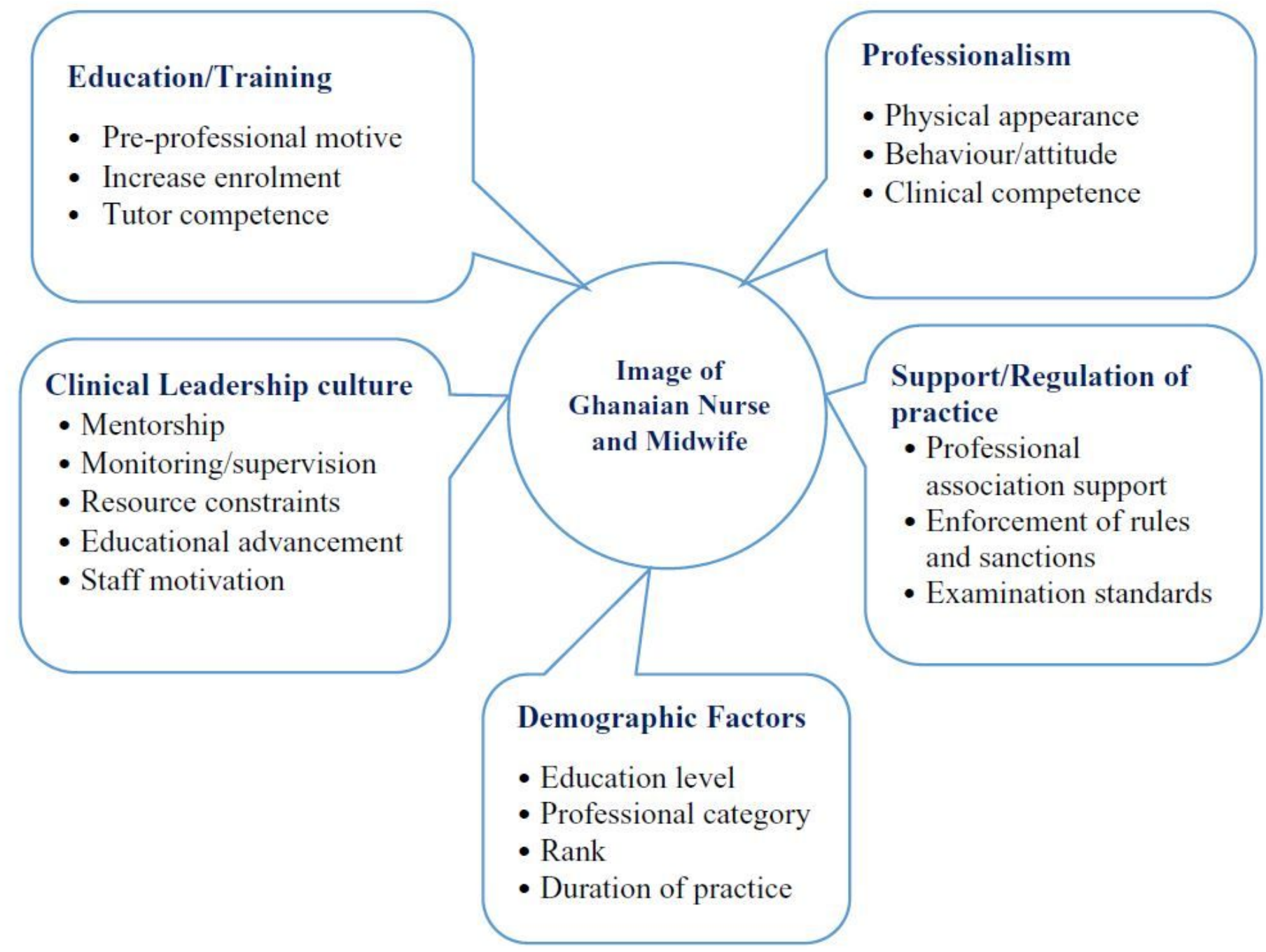

Figure 1

Determinants of image of nurses and midwives 


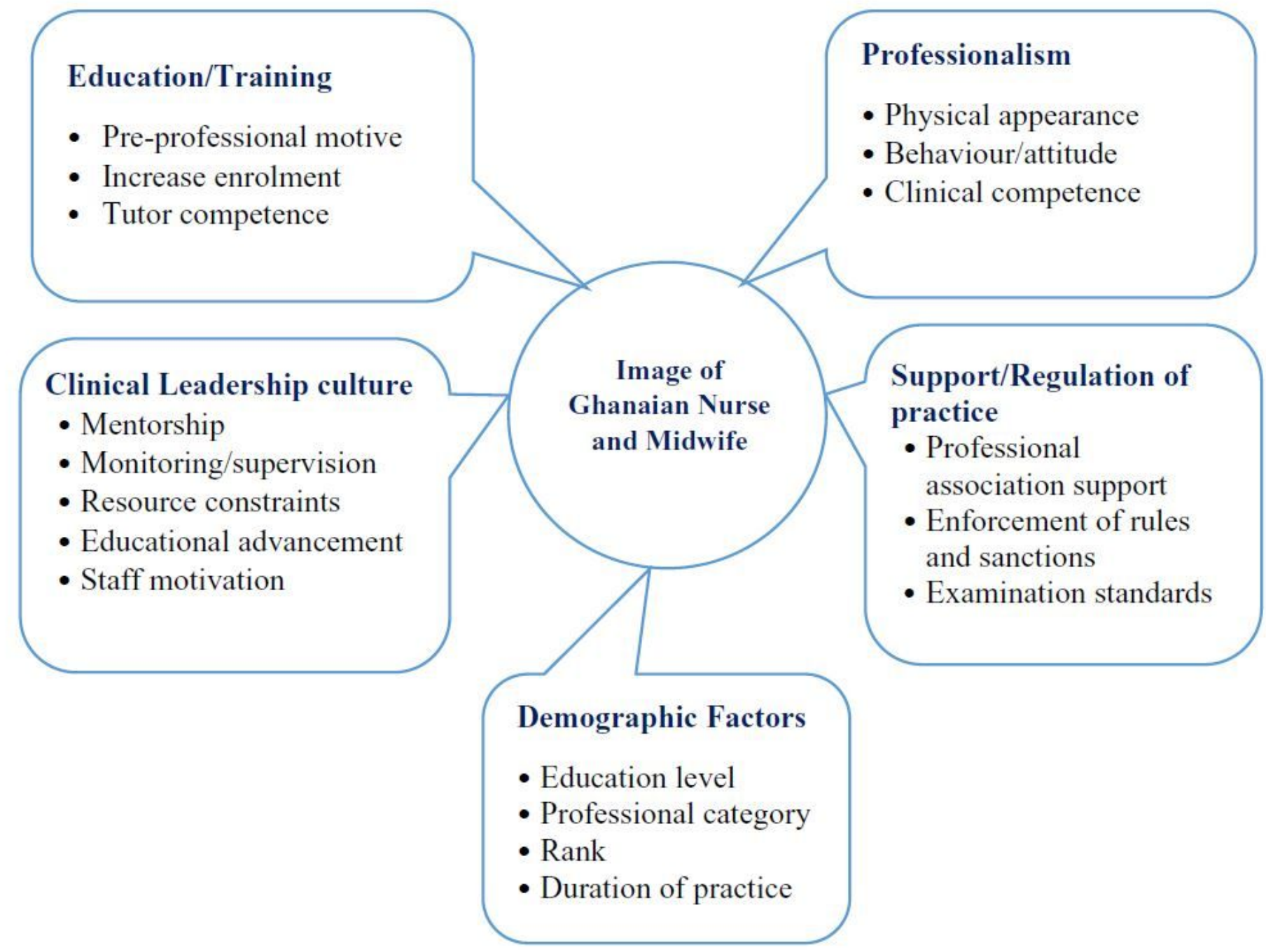

Figure 1

Determinants of image of nurses and midwives

\section{Supplementary Files}

This is a list of supplementary files associated with this preprint. Click to download.

- Research.Instrument.docx

- Research.Instrument.docx 